\title{
Static and Dynamic Properties of Block-Copolymer Solution in Poor Solvent I. A Light Scattering Study on Solutions in Weakly-Selective Solvents
}

\author{
Takamasa Azuma, Onkar S. TyAGI ${ }^{\dagger}$, and Takuhei Nose ${ }^{\dagger \dagger}$ \\ Department of Polymer Chemistry, Tokyo Institute of Technology, \\ Ookayama, Meguro-ku, Tokyo 152-8552, Japan
}

(Received August 3, 1999)

\begin{abstract}
By means of static and dynamic light scattering, we investigate competitive behavior of association and liquid-liquid phase separation in solutions of two diblock copolymers in weakly-selective poor solvent. Near the critical point of liquid-liquid phase separation, the copolymer consisting of chemically similar blocks poly(dimethylsiloxane) (PDMS) and poly(1,1,3,3-tetramethyldisiloxanylethylene) (PTMDSE) shows very weak association, exhibiting the ordinary critical behavior of 3-dimensional (3D) Ising model, but the copolymer consisting of chemically dissimilar blocks of PDMS and polystyrene (PS) shows two modes in static and dynamic light scattering, showing increasing association as well as the critical concentration fluctuation with the liquid-liquid phase separation being approached. The static critical behavior obeys the 3D Ising model as in PDMS-block-PTMDSE, while the copolymer associations bring about unique dynamic properties in poor solvent different from those of homopolymer solutions.
\end{abstract}

KEY WORDS Block Copolymer/Solution / Critical Phenomena / Association / Light Scattering /

Structure and phase behavior of block copolymer solutions have been attracting increasing research interests recently as they exhibit a wide variety of static and dynamic properties, depending on solvent quality and selectivity, and segregation-strength between blocks. In a good solvent for both blocks, the solution usually shows disorder-to-order transition with increasing concentration, ${ }^{1,2}$ while in a selective solvent, association of macromolecules takes place, leading to formation of micelles of various types in dilute solution. ${ }^{3}$ On the other hand, in a non-(or weakly-)selective poor solvent, the solution may show liquid-liquid phase separation with critical phenomena, similar to or different from ordinary polymer solutions. ${ }^{4}$ In a (strongly-)selective solvent, the solution exhibits gelation as the solvent is getting poorer. ${ }^{5-7}$ The situation becomes simple or complex depending upon the result of their competition.

By mainly using static and dynamic light scattering techniques we have recently found that in real copolymer solutions in poor solvents, one can observe competitive ordering processes of association, phase separation and gelation. This paper is part of a series of studies dealing with such competitive phenomena in different types of copolymer/solvent systems aiming to elucidate structure and phase behavior of copolymer solutions. We deal with two types of systems: block copolymer solutions (1) in selective poor solvents and (2) in weaklyselective poor solvents. In the solution (1), the three affairs may competitively occur depending on temperature and concentration, while in the solution (2), one can observe critical phenomena with intermolecular association.

In the present paper we report the results of combined static and dynamic light scattering study on the solution (2), i.e., solutions of two diblock copolymers of poly(dimethylsiloxane) (PDMS), one with a chemically similar block of poly(1,1,3,3-tetramethyldisiloxanylethylene) ( $\mathrm{P}$ TMDSE) and another with a chemically dissimilar block of polystyrene (PS), in weakly-selective solvents. These polymer solutions may exhibit liquid-liquid phase separation, that give, respectively, weak- and strongassociations of macromolecular chains during critical phenomena leading to the phase separation.

\section{EXPERIMENTAL}

\section{Materials}

The samples of diblock copolymers PDMS- $b$-PTMDSE and PS- $b$-PDMS were prepared by sequential anionic polymerization of dimethylsiloxane with 1,1,3,3-tetramethyldisiloxanethylene and styrene, respectively. Characteristics of these polymers are listed in Table I .

\section{Sample Solutions}

All solvents were analytical grade and were used without further purification. The selection of solvent quality for the purpose of this study was done by investigation of the cloud points $\left(T_{\mathrm{cl}}\right)$ of respective homopolymers (PDMS, PTMDSE, and PS) having similar molecular weight ranges.

For System I. PDMS-b-PTMDSE/Phenatol. Ethyl phenyl ether (phenatol) was selected because it is a poor solvent for both blocks of the copolymer but is selectively poorer for PDMS than for PTMDSE. At a fixed polymer

Table I. Characteristics of block copolymer samples

\begin{tabular}{ccccc}
\hline Sample code & \multicolumn{2}{c}{$M . W$. of blocks } & $M . W$. & $M_{w} / M_{n}$ \\
\hline PDME- $b$-PTMDSE $^{\text {a }}$ & PDMS & PTMDSE & & \\
& 12400 & 12000 & 24400 & 1.19 \\
\hline PS- $b$-PDMS $^{\mathrm{b}}$ & PS & PDMS & & \\
& 7800 & 3200 & 11000 & 1.18 \\
\hline
\end{tabular}

a Molecular weights (M.W.) are the weight-average molecular weight $\left(M_{w}\right)$ determined by size-exclusion chromatography (SEC) with PDMS-standard. The composition of PDMS/PTMDSE was measured by ${ }^{1} \mathrm{H}$ NMR. ${ }^{b}$ Molecular weights are the numberaverage molecular weight $\left(M_{w}\right)$ determined by PS-standard SEC.

\footnotetext{
${ }^{\dagger}$ Present address : Indian Institute of Petroleum (CSIR), Dehradun 248005, India.

${ }^{\dagger+}$ To whom correspondence should be addressed.
} 
concentration ( $15 \mathrm{wt} \%$ ) in this solvent, a PDMS sample of $M_{w}=2.20 \times 10^{4}$ showed $T_{\mathrm{cl}}$ at about $53^{\circ} \mathrm{C}$ while a PTMDSE sample of $M_{w}=2.91 \times 10^{4}$ showed $T_{\mathrm{cl}}$ at about $31^{\circ} \mathrm{C}$. It was also found that this PDMS- $b$-PTMDSE exhibited a disorder state in bulk. These observations indicated PDMS- $b$-PTMDSE / phenatol system to be a case of a weak-segregation (chemically similar) block copolymer in a weakly-selective (poor) solvent, suitable for present study.

For System II. PS-b-PDMS/ (n-Octane +Cyclohexanol). Mixed $n$-octane+cyclohexanol solvent was selected because it was observed that cyclohexanol was a poor solvent for both blocks of copolymer but was selectively poor for PDMS, while the goodness of solvent increased selectively for PDMS when $n$-octane was mixed in it. The optimum composition of $n$-octane in mixed solvent needed to achieve the quality of a weakly (or non-) selective solvent was determined by measuring $T_{\mathrm{cl}}$ of two samples of homopolymers PS and PDMS, both having nearly identical molecular weights $\left(M_{w} \cong 10000\right)$, at the fixed concentration of $15 \mathrm{wt} \%$ polymer in the mixed solvent of different compositions. The results are illustrated by plots of $T_{\mathrm{cl}} v s$. the mixed solvent composition in Figure 1, showing that: (i) in pure cyclohexanol, $T_{\mathrm{cl}}$ of PDMS is about $118^{\circ} \mathrm{C}$ while that of PS was about $51^{\circ} \mathrm{C}$, and (ii) with increasing concentration of $n$-octane in the solvent system, the decrease in $T_{\mathrm{cl}}$ of PDMS is substantial and that of PS is marginal, so that $T_{\mathrm{cl}}$ 's of both homopolymers become almost equal (about $38^{\circ} \mathrm{C}$ ) at 40 wt\% $n$-octane composition in the mixed solvent. Earlier investigations on a blend of low-molecular weight PS and PDMS have shown that a pair of PS and PDMS is a strongly immiscible one, ${ }^{8}$ and this PS- $b$-PDMS is in an ordered state in bulk. ${ }^{9}$ These data indicate that the PS- $b$ PDMS solution in $40: 60 \mathrm{wt} \%$ mixture of $n$-octane + cyclohexanol may behave like a strongly-segregated (chemically dissimilar) copolymer in a weakly (or non-) selective poor solvent for the purpose of present study.

\section{Coexisting Curve Measurements}

System I produced two transparent, demixed-phases upon phase separation. Its coexisting curve was determined by using a specially-designed laboratoryconstructed differential refractometer. The details of apparatus and experimental procedure have been described elsewhere. ${ }^{8}$ System II was also phase separated into two demixed-phases, but it took much longer time to obtain a clear boundary between them. This made it difficult to obtain a complete coexisting curve in a reasonable time.

\section{Static Light Scattering Measurements}

The light scattering measurements were carried out with a home-built apparatus equipped with a $\mathrm{He}-\mathrm{Ne}$ laser $(\lambda=633 \mathrm{~nm})$ and photon detector (ALV/SO-SIPD). The sample tube was immersed in an index-matching oil bath thermoregulated to within $\pm 0.05^{\circ} \mathrm{C}$. Optically clean solutions were prepared as follows. A dilute solution of copolymer in benzene was filtered through 0.22 $\mu \mathrm{m}$ filters (Millipore) into a dedusted light scattering cell from which benzene was subsequently evaporated (first under normal conditions and then in a vacuum oven at $80^{\circ} \mathrm{C}$ until all benzene was removed). Next, an appropri-

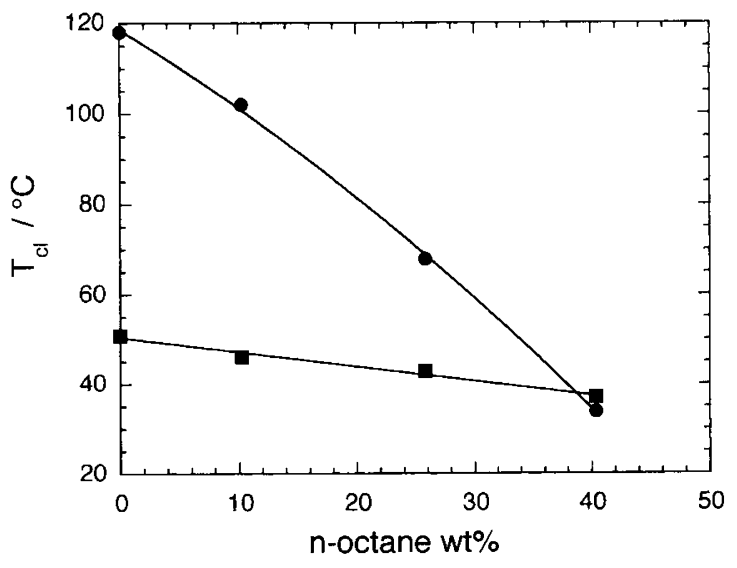

Figure 1. Dependencies of cloud points $T_{\mathrm{cl}}$ of PS( $\left.\square\right)$ and PDMS (O) on composition of $n$-octane+cyclohexanol mixed solvent at fixed polymer concentration $(15 \mathrm{wt} \%)$ and molecular weight $\left(M_{w} \cong\right.$ 10000). Solid lines are guides for the eye.

ate amount of the solvent was filtered directly into this cell to give desired concentration of solution and the cell was sealed after replacing the air in dead space in the cell by $\mathrm{N}_{2}$ gas. The solution was allowed to equilibrate for sufficiently long duration before measurements. The scattered light intensity $(I)$ from the solution was measured at different scattering angles $(\theta)$ in the range of 30 ${ }^{\circ} \mathrm{C}$ to $120^{\circ} \mathrm{C}$, at various temperatures from about $100^{\circ} \mathrm{C}$, where the solutions were expected to be free from larger aggregates, down to the critical temperature. The integrated intensity $I(q)$ of scattered light were obtained as functions of the wave number $(q)$ and temperature. Here, $q=(4 \pi n / \lambda) \sin (\theta / 2)$ with $n$ and $\lambda$ being the refractive index of sample solution and the wavelength of incident beam, respectively. The inner diameter of sample tubes was $4.2 \mathrm{~mm}$, and the maximum attenuation by turbidity was estimated to be about $10 \%$, except at the temperature closest to the stability limit in PDMS- $b$-PTMDSE solution. In most cases, the attenuation was estimated to be less than a few percents. The correction of multiple scattering must be of the same order of that of the attenuation. The effects of attenuation and multiple scattering were negligible for the purpose of the present study. Thus no correction of the scattered light intensity was made for attenuation and multiple scattering.

\section{Quasi-elastic Light Scattering Measurements}

The homodyne intensity autocorrelation function of scattered light was measured with an ALV-5000 multiple- $\tau$ digital correlator. Using the assumption of homodyne scattering and the Siegert relation ${ }^{10}$ (eq 1), the electric-field correlation functions $g^{(1)}(q, t)$ was calculated.

$$
g^{(2)}(q, t)=A\left[1+\beta\left|g^{(1)}(q, t)\right|^{2}\right]
$$

where $A$ and $\beta$ are constants. In cases of the unimodal decay of $g^{(1)}(q, t)$, the second cumulant method was used to calculate the decay rate $\Gamma$. In cases of the bimodal decay, the decay function of $g^{(1)}(q, t)$ was analyzed by the Laplace inversion program CONTIN to separate the two modes and evaluate respective decay rates and ampli- 


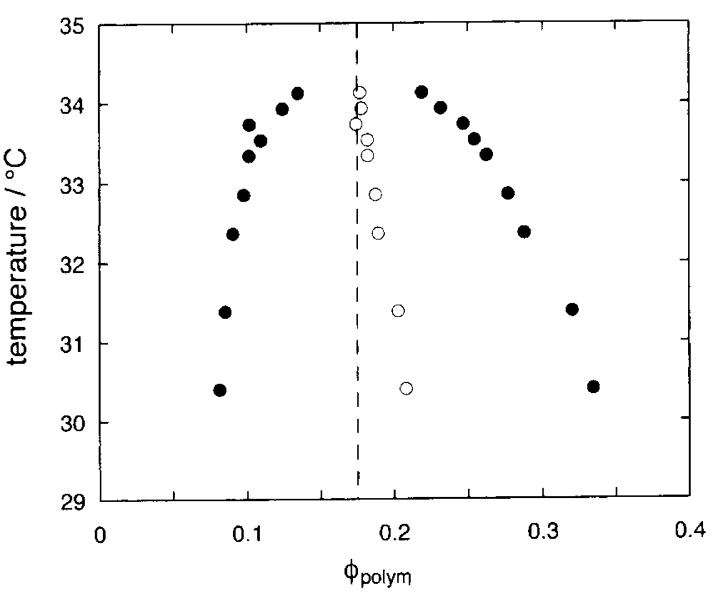

Figure 2. Coexistence curve of PDMS- $b$-PTMDSE solution in phenetol. Filled circles are plots of temperature vs. polymer volume fractions $\phi_{\text {mym }}$ of coexisting phases. Hollow circles : diameter values defined by a half-of-the sum of concentrations of demixed phases. Dashed line : estimated critical concentration.

tudes.

\section{RESULTS AND DISCUSSION}

\section{System I. PDMS-b-PTMDSE / Phenatol}

Coexisting Curves. This system upon phase separation produced two well-defined demixed phases, both transparent and without any inhomogeneity, resulting in the coexisting curve similar to ordinary liquid-liquid mixtures. Figure 2 shows the coexisting curve represented as the volume fraction $\phi$ of PDMS- $b$-PTMDSE in coexisting phases as a function of temperature. Fitting the measured concentration difference $\Delta \phi$ in volume fractions between two phases at various temperatures into the equation ${ }^{11}$ :

$$
\Delta \phi \sim\left[\left(T-T_{\mathrm{c}}\right) / T\right]^{;}
$$

gave the values of critical temperature $T_{\mathrm{c}}=34.27^{\circ} \mathrm{C}$ and critical exponent $\beta=0.34_{2}$. The value of $\beta$ is close to that $(\beta=0.33)$ of 3 -dimensional (3D) Ising model, ${ }^{11}$ showing that this liquid-liquid phase separation is of an ordinary binary liquid mixture or a simple fluid. The diameter of the coexisting curve at $T_{\mathrm{c}}$ gave the critical concentration $\left(\phi_{c}\right)$ to be 17.5 vol\% of PDMS- $b$-PTMDSE in phenatol solution, where the diameter is the simple average $\left(\phi^{\prime}+\right.$ $\left.\phi^{\prime \prime}\right) / 2$ of the concentrations, $\phi^{\prime}$ and $\phi^{\prime \prime}$, of the two coexisting phases. Following these results, detailed investigations on static and dynamic properties of this system were carried out on the solution with the fixed polymer concentration of $17.5 \mathrm{vol} \%$, i.e., at $\phi_{c}$, and at temperatures above $T_{\mathrm{c}}$ in one-phase region.

Static Critical Behavior in One Phase Region. For the PDMS- $b$-PTMDSE/phenetol system near the critical point the wave number $q$-dependence of $I(q)$ is well described by the Ornstein-Zernike equation :

$$
I(0) / I(q)=1+q^{2} \xi^{2}
$$

as indicated by good linear relation between $I^{-1}(q)$ and $q^{2}$ shown in Figure 3. This suggests that there is no as-

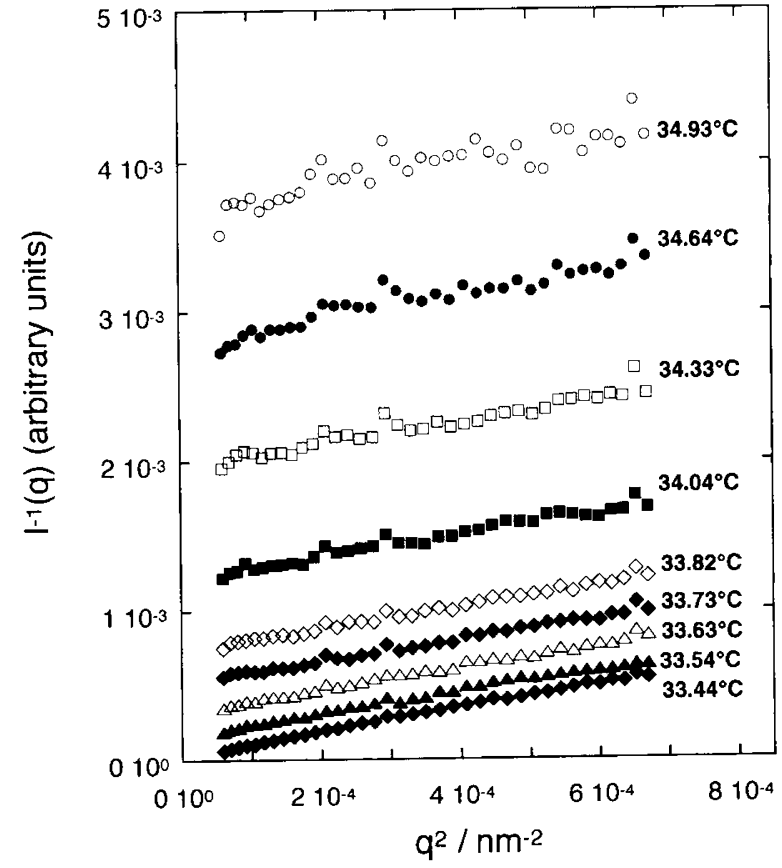

Figure 3. Ornstein-Zernik plots for the 17.5 vol\% PDMS- $b$ PTMDSE/phenetol solution at indicated temperatures.

sociation or that the association is very weak in this system. Hence, the correlation length $\xi$ and $I^{-1}(0)$ were determined by least squares fittings of data to eq 3 .

The inverse osmotic compressibility $(\mathrm{d} \pi / \mathrm{d} c)_{\mathrm{T}}$ or inverse susceptibility $S_{\mathrm{T}}{ }^{-1}$ was evaluated by $T / I(0)$ in an arbitrary scale. The obtained values of $\xi$ and $S_{\mathrm{T}}{ }^{-1}$ show good linearity with the reduced temperature $\left(T-T_{\mathrm{s}}\right) / T$, in double-logarithmic plots as shown in Figure 4, suggesting that the quantities $\xi$ and $S_{\mathrm{T}}{ }^{-1}$ are well described by power laws. The values of spinodal temperature $\left(T_{\mathrm{s}}\right)$, critical amplitude $\left(S_{0}\right)$ and critical exponent $(\gamma)$ for susceptibility were evaluated by least-squares fittings of the data near the stability limit to the relation:

$$
S_{\mathrm{T}}=S_{0}\left|T_{\mathrm{s}} / T-1\right|^{-\gamma}
$$

Using thus evaluated $T_{\mathrm{s}}\left(=34.01^{\circ} \mathrm{C}\right)$, the critical exponent $v$ for the correlation length was determined by fitting $\xi$ to the relation:

$$
\xi=\xi_{0}\left|T_{\mathrm{s}} / T-1\right|^{-\nu}
$$

where $\xi_{0}$ is the critical amplitude. The fitting results (shown by solid lines in Figure 4) well describe the experimental results. The obtained values of critical exponents $\gamma=1.25$ and $\nu=0.62$ are close to the values expected for the 3D Ising model $(\gamma=1.24 \text { and } \nu=0.63)^{11}$ and indicate nothing different from normal static critical behavior of an ordinary binary fluid mixture.

Dynamic Critical Behavior. The experimental $g^{(1)}(q$, $t$ ) showed a single exponential decay. The decay rate $\Gamma$ decreases with the stability limit being approached (i.e., as $\xi$ increases), showing the so-called critical slowdown. In the hydrodynamic region of $q \xi<1$ near the stability limit, the diffusion coefficient $D=\Gamma / q^{2}$ is independent of $q$ and should follow the equation : 


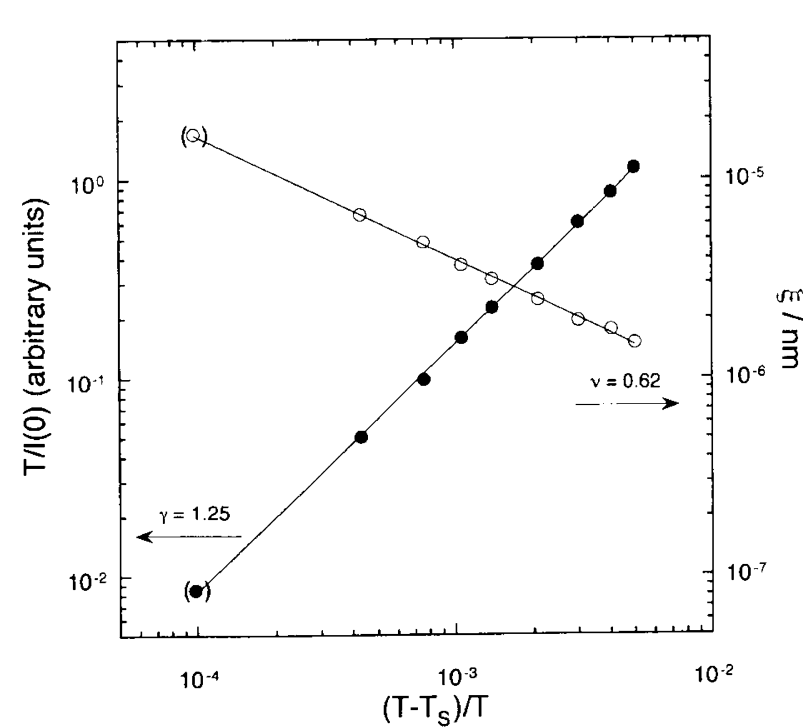

Figure 4. Double-logarithmic plots of $T / I(0)$ and $\xi$ against ( $T$ $T_{s} / T$. Straight lines represent the least-squares fittings of power laws. Plots in brackets have not sufficient precision for temperature.

$$
D \sim 1 / \xi \sim\left|T_{\mathrm{s}} / T-1\right|^{-v}
$$

Figure 5 shows the double logarithmic plots of $D$ against $\left(T-T_{\mathrm{s}}\right) / T$, which indicates that the relation of eq 6 holds. Namely, the plots were approximated by eq 6 with $\nu=$ 0.63 , although the dependence of $D$ on $\left|\left(T_{\mathrm{s}} / T\right)-1\right|$ becomes weak as $T_{\mathrm{s}}$ is approached. This suggests that the dynamics exhibits the ordinary critical behavior in fluid systems. (Details of the dynamic behavior will be discussed in a forthcoming paper of this series.)

In conclution, the PDMS- $b$-PTMDSE/phenatol solution, which is a weakly-segregated copolymers in weaklyselective poor solvent, shows an ordinary liquid-liquid phase separation, exhibiting ordinary critical behaviors in static and dynamic properties, as expected.

\section{System II.PS-b-PDMS / (n-Octane +Cyclohexanol)}

Static Behavior. The scattered-light intensity shows a complex dependence on both $q$ and $T$ (Figures 6 and 7) in contrast to the system I. At $100^{\circ} \mathrm{C}$ the $I^{-1}(q)$ vs. $q^{2}$ plots are reasonably flat at high $I^{-1}$-values (at very low intensities) except at very low angles, indicating a very weak aggregation effect, if any (Figure 6a). At $T<80^{\circ} \mathrm{C}$ the scattered-light intensity increases even at higher scattering angles and shows a strong $q$-dependence particularly at $T<60^{\circ} \mathrm{C}$ (Figure $6 \mathrm{~b}$ ). These results indicate that upon cooling the association-free solution, the aggregation of macromolecular chains begins at about $80^{\circ} \mathrm{C}$ and the phenomenon becomes stronger with decrease in temperature particularly below $60^{\circ} \mathrm{C}$ resulting in curved Zimm (Ornstein-Zernike) plots. The bending of curves and simultaneous shifting of their plateau to higher $q$ reveals that the measured $I(q)$ has at least two components, designated here as $I_{\mathrm{m}}$ and $I_{\mathrm{a}}$, arising from concentration fluctuations and large size structures (or aggregates formed during cooling process) respectively. That is, one may put

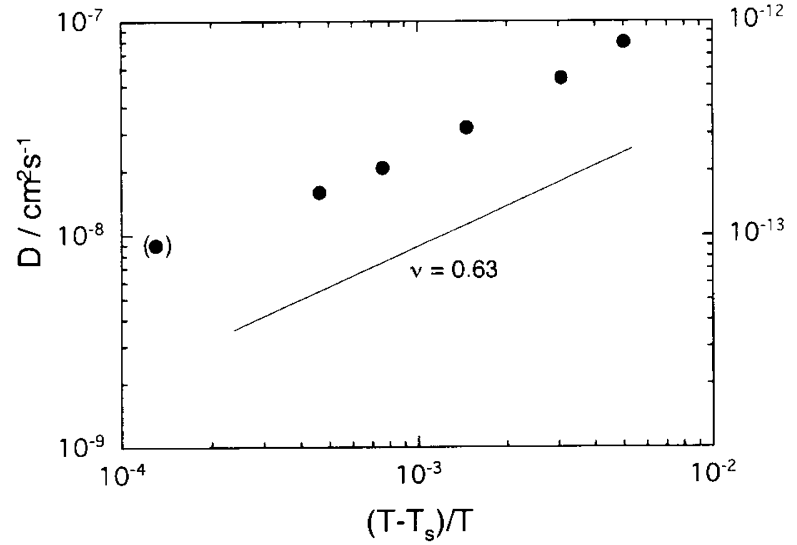

Figure 5. Double-logarithmic plots of the diffusion coefficient $D$ against $\left(T-T_{\mathrm{s}}\right) / T$. Straight line indicates the predicted power of eq 6 . A plot in bracket has not sufficient precision for temperature.

$$
I(q)=I_{\mathrm{m}}(q)+I_{\mathrm{a}}(q)
$$

As the temperature approaches the stability limit, one observes very large increase in the scattered-light intensity with disappearance of the bending of OrnsteinZernike plots which became almost straight at $T \sim T_{\mathrm{s}}$, a pattern characteristic of critical fluctuation in ordinary liquid mixtures. (Figure 7) This indicates that the concentration fluctuation is the dominant contribution to measured $I(q)$ at critical phase separation, to the extent that at $T \sim T_{\mathrm{s}}, I_{\mathrm{m}}>>I_{\mathrm{a}}$ and at least at higher scattering angles $I_{\mathrm{m}}(q)$ may be taken equal to the measured $I(q)$ value. The $I_{\mathrm{m}}$ and $I_{\mathrm{a}}$ contributions to measured $I(q)$ and their $q$ - and $T$-dependences are estimated as described below.

The $I_{\mathrm{m}}(q)$ are first determined by the following procedures $1-3$. The intensity $I_{\mathrm{m}}(q)$ is assumed to be expressed by the Ornstein-Zernike type equation, eq 8 .

$$
I_{\mathrm{m}}(q)=\frac{I_{\mathrm{m}}(0)}{1+\xi_{\mathrm{m}}^{2} q^{2}}
$$

1. At temperature below $34^{\circ} \mathrm{C}$ closest to the stability limit, $I_{\mathrm{m}}(q)$ values can be equated with $I(q)$ because of small contribution of $I_{\mathrm{a}}(q)$, which is expressed by eq 8 .

2. It was also the case at a little higher temperatures (34, 35,36 , and $38^{\circ} \mathrm{C}$ ) close to $T_{\mathrm{s}}$ for $I(q)$ measured at higher scattering angles, so that in this temperature range, $I_{\mathrm{a}}$ contribution is subtracted by the following way. First $\xi_{\mathrm{m}}$ is determined by fitting to eq 8 from the data at higher angles assuming that $I_{\mathrm{a}}=0$. Then, the bimodal fitting with eq 7 is conducted for the whole range of $q$ observed with fixed values of $\xi_{\mathrm{m}}$ that have been obtained by the first procedure. In this case, we assume eq 8 and approximately express $I_{\mathrm{a}}(q)$ as

$$
I_{\mathrm{a}}(q)=\frac{I_{\mathrm{a}}(0)}{1+\xi_{\mathrm{a}}^{2} q^{2}}
$$

for the experimental $q$-range. Obtained $I_{\mathrm{m}}(0)$ and $\xi_{\mathrm{m}}$ can give $I_{\mathrm{m}}(q)$ through eq 8 . Although the accuracy of determination of $\xi_{\mathrm{m}}$-value is not good, $I_{\mathrm{m}}(0)$ is not affected by small values of $\xi_{\mathrm{m}}$.

3. For temperature higher than $38^{\circ} \mathrm{C}, I_{\mathrm{m}}$ are so small that we cannot determine $\xi_{\mathrm{m}}$, but putting $\xi_{\mathrm{m}}=0$, one 

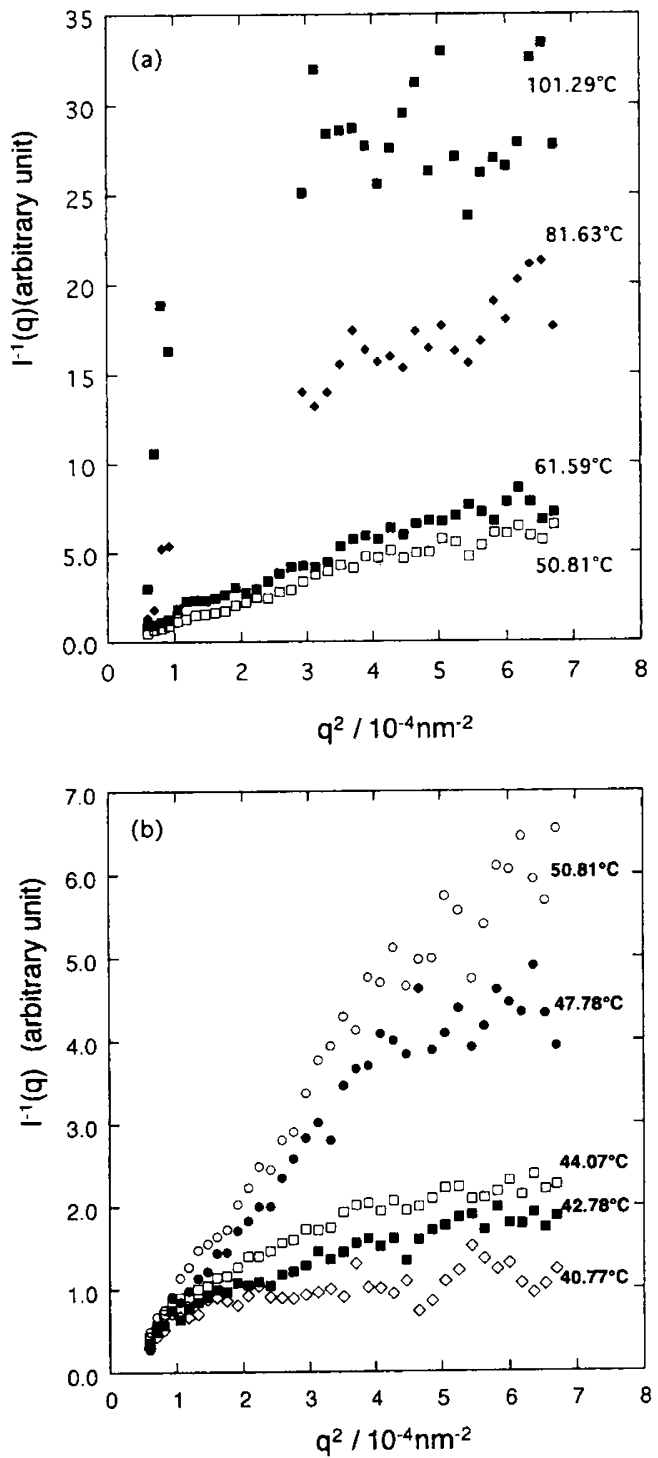

Figure 6. Ornstein-Zernike plots of $I^{-1}(q)$ against $q^{2}$ for $15.6 \mathrm{wt}$ $\%$ PS- $b$-PDMS solution in $n$-octane+cyclohexanol $(40: 60 \mathrm{wt} \%)$ at temperatures indicated (a) far above and (b) approaching the stability limit of solution.

can reasonably determine $I_{\mathrm{m}}(0)$ by the same fitting. It should here be noted that the fitting is not so good that the evaluated $I_{\mathrm{a}}(0)$ and $\xi_{\mathrm{a}}$ do not imply the real zero-angle scattering and the correlation length, respectively, since eq 9 is used just for an approximate expression for the present purpose of subtracting $I_{\mathrm{m}}-$ contribution.

Now we have $I_{\mathrm{m}}(q)$, then we calculate $I_{\mathrm{a}}(q)$ by subtracting $I_{\mathrm{m}}(q)$ from observed total intensity, i.e., $I_{\mathrm{a}}(q)=I(q)-$ $I_{\mathrm{m}}(q)$ for the whole range of temperature.

(i) Contribution of Concentration Fluctuations and Critical Behavior. At the closest temperature range near the stability limit (below $34^{\circ} \mathrm{C}$ ), the $I(q)$ follows the Ornstein-Zernike type relation, yielding the values of $I_{\mathrm{m}}(0)$ and $\xi \mathrm{m}$ for critical concentration fluctuations. These values allow us to make plots of inverse susceptibility $(=T /$ $\left.I_{\mathrm{m}}(0)\right)$ and $\xi$ against reduced temperature to evaluate the spinodal temperature $T_{\mathrm{s}}$ and the critical exponents, $\gamma$ and $\nu$, by the least-square fittings to eq 4 and 5 , which are shown in Figure 8. The linearity of these plots dem-

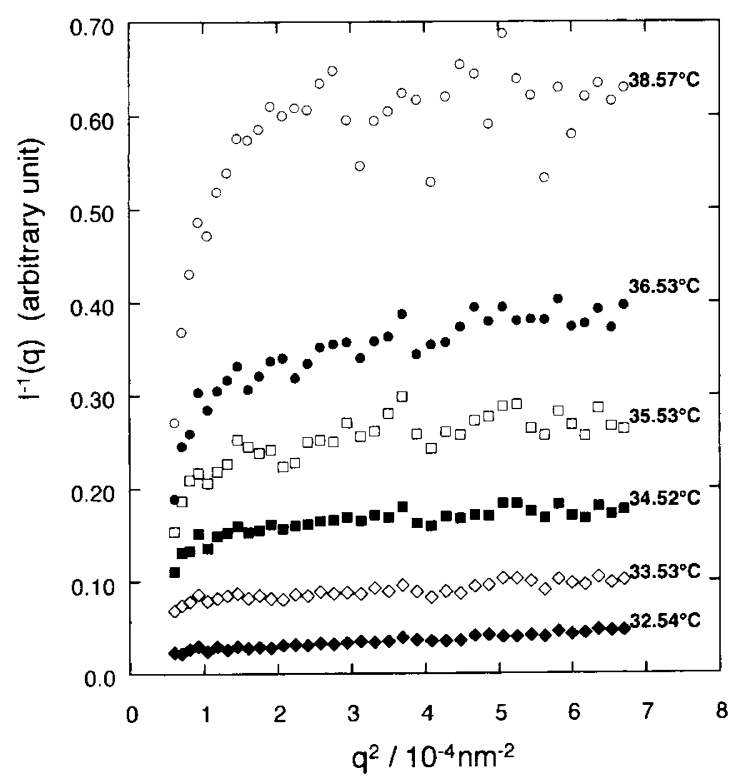

Figure 7. Ornstein-Zernike plots of $I^{-1}(q)$ against $q^{2}$ for $15.6 \mathrm{wt}$ $\%$ PS- $b$-PDMS solution in $n$-octane+cyclohexanol $(40: 60 \mathrm{wt} \%)$ at temperatures indicates near the stability limit of solution.

onstrates that these parameters follow the power law. The least-squares analyses gave $T_{\mathrm{s}}=31.94^{\circ} \mathrm{C}, \gamma=1.26_{8}$, and $\nu=0.62_{5}$. These values confirm that the system obeys the 3D Ising model. That is, it seems to behave like an ordinary fluid phase separation, as far as static properties are concerned.

$I_{\mathrm{m}}(0)$ and $\xi_{\mathrm{m}}$ at higher temperatures are plotted against reduced temperature in Figure 9. The upward trend of the $I_{\mathrm{m}}(0)$-plots in the present case may suggest that large aggregates are growing in solution as the temperatures is lowering to the critical point. The accuracy of determination of $\xi \mathrm{m}$ value at the higher temperatures of $38^{\circ} \mathrm{C} \geq T \geq 34^{\circ} \mathrm{C}$ is not so good that nothing can be commented on it.

(ii) Contribution of Aggregation. The $q$-dependence of $I_{\mathrm{a}}$ at different temperatures is shown in Figure 10a. At temperatures of $60^{\circ} \mathrm{C}<T<100^{\circ} \mathrm{C}, I_{\mathrm{a}}$ is almost zero, showing no substantial aggregation. Around $60^{\circ} \mathrm{C}, I_{\mathrm{a}}(q)$ having upturn at lower values of $q$ appears and starts to grow with decreasing temperature. (At lower temperatures of $T<40^{\circ} \mathrm{C}, I_{\mathrm{a}}$ was not estimated accurately due to large contribution of $I_{\mathrm{m}}(q)$.) As is shown in Figure 10b, the double-logarithmic plots of $I_{\mathrm{a}}(q) v s . q^{2}$ are linear (the slope of fitted line indicated are around unity) and almost parallel with each other, indicating an increase in $I_{\text {a }}$ value with decrease in temperature but no change in its trend with $q$. That is, the number of aggregates and / or their sizes increase with decrease in temperature. The power law, i.e., the linearity of these plots probably suggests formation of a fractal structure.

In Figure 11, are shown the temperature-dependencies of $I_{\mathrm{a}} / T$ and its fraction, $I_{\mathrm{a}} / I$, in the total intensity $I$, at selected two scattering angles. The trend indicates that although the aggregation is increasing with decrease in temperature, yielding higher values for $I_{a} / T$, their relative contribution to measured $I(q)$ drops sharply on approaching the stability limit, in other words, irrespective of growing inhomogeneity in solution the critical fluctuation represented by $I_{\mathrm{m}}$ dominates aggregations near the 


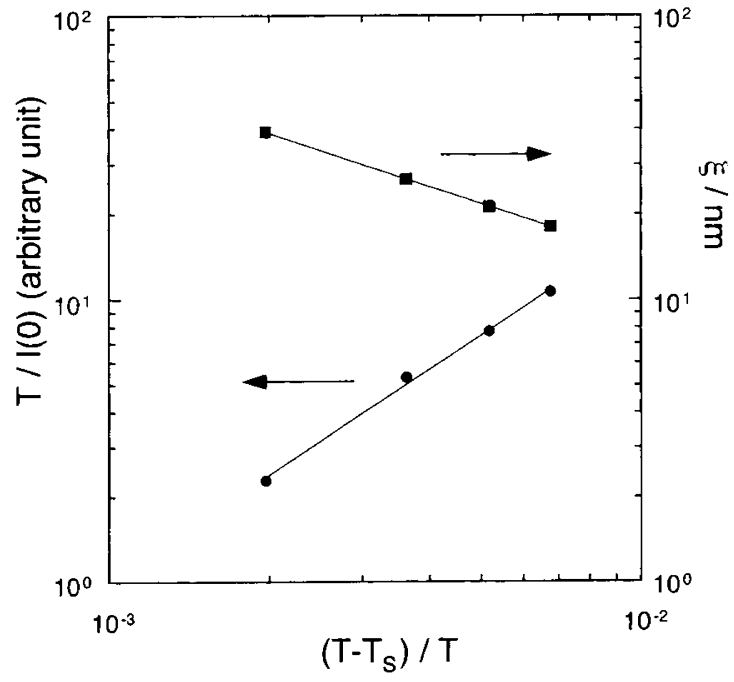

Figure 8. Double-logarithmic plots of inverse isothermal susceptibility $T / I(0)(\bigcirc)$ and correlation length $\xi(\boldsymbol{\square})$ against the reduced temperature for the $15.6 \mathrm{wt} \%$ PS- $b$-PDMS solution in a $40: 60 \mathrm{wt}$ $\% n$-octane+cyclohexanol mixed solvent. Straight lines represent the fitting results to the power law.

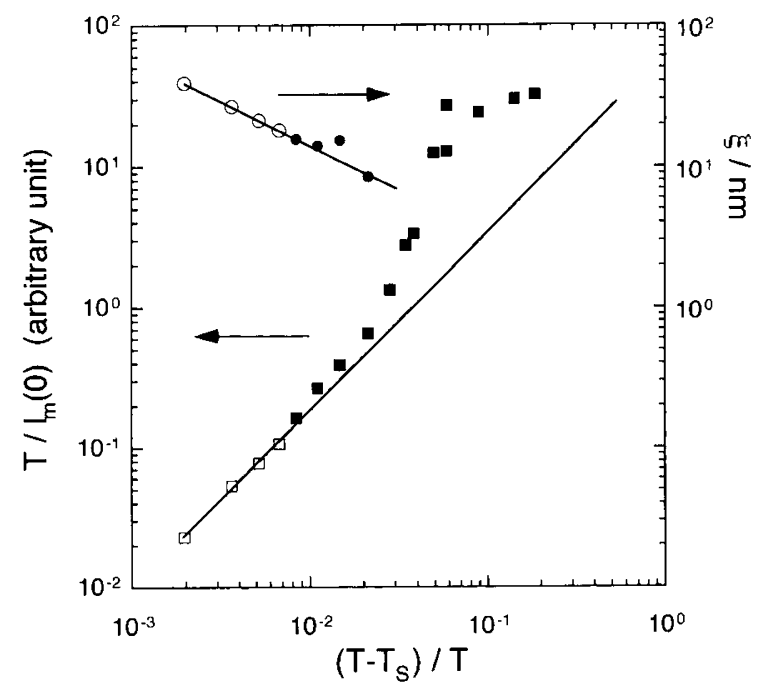

Figure 9. Variation in the amplitude $I_{\mathrm{m}}(0)$ and correlation length $\xi$ with the reduced temperature $\left(T-T_{\mathrm{s}}\right) / T$ at higher temperatures, along with those in the vicinity of stability limit $T_{\mathrm{s}}$. Solid symbols are for higher temperatures, and open symbols are plots near $T_{\mathrm{s}}$. Straight lines represent the fitting lines near $T$ drawn in Figure 8.

stability limit. This observation suggests that $I_{\mathrm{a}}$ does not strongly couple with $I_{\mathrm{m}}$.

Thus the above static properties observed by light scattering on PS- $b$-PDMS/( $n$-octane + cyclohexanol) do not show a large deviation from ordinary critical phase separation behavior similar to the behavior of System I, although unlike System I this system showed a strong aggregation at temperatures much above the critical point.

Dynamic Behavior as Observed by Dynamic Light Scattering. A typical example of the correlation function $g^{(2)}(q, t)$ together with its CONTIN analysis is shown in Figure 12. The intensity correlation functions are characterized by two distinct relaxation processes with relaxation rates and amplitudes that have characteristic dependencies on temperature and scattering
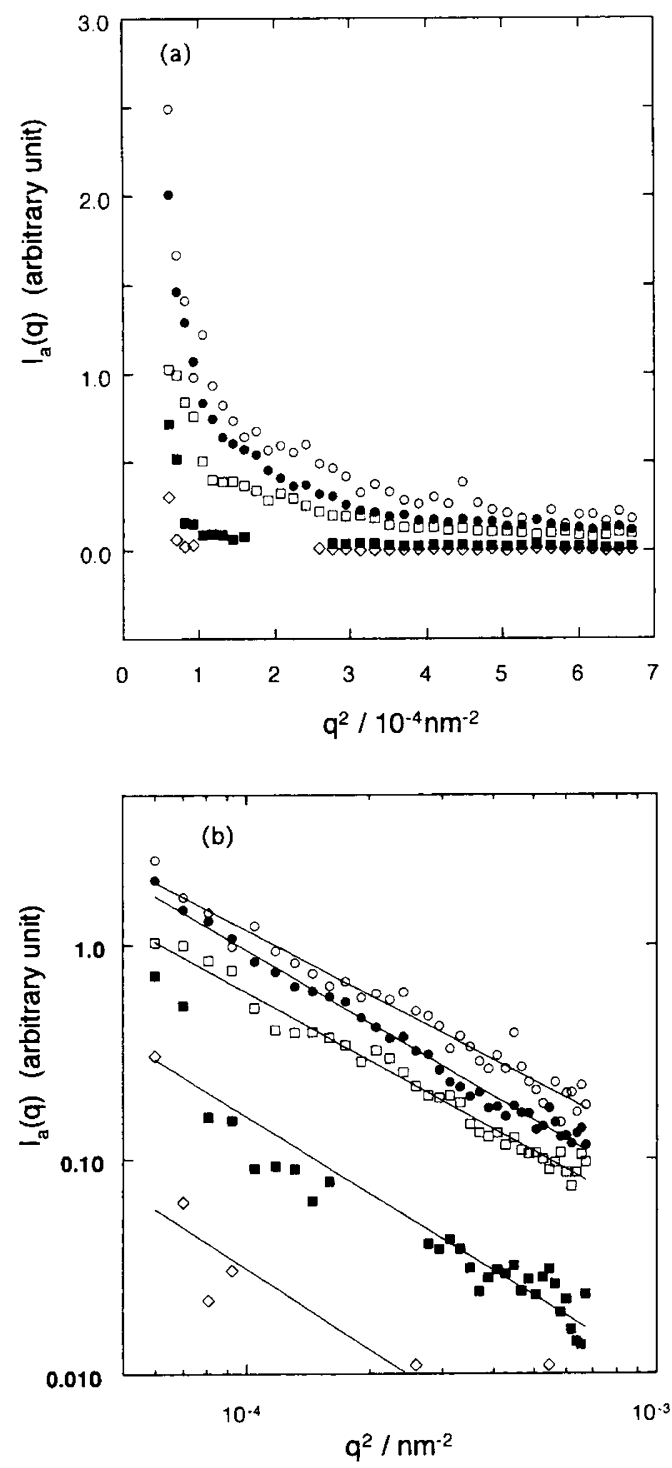

Figure 10. Plots of $I_{\mathrm{a}}$ against $q^{2}$ for the $15.6 \mathrm{wt} \%$ PS- $b$-PDMS solution in the $40: 60 \mathrm{wt} \% n$-octane+cyclohexanol mixed solvent at different temperatures. (a), linear scale plots; (b), log-log plots (straight lines represent the least-squares fittings.) $(\bigcirc), 42^{\circ} \mathrm{C} ;(\bigcirc)$, $50^{\circ} \mathrm{C} ;(\square), 60^{\circ} \mathrm{C} ;(\square), 80^{\circ} \mathrm{C} ;(\diamond), 100^{\circ} \mathrm{C}$.

wave number, as shown in Figures 13 and 14, respectively.

Temperature-dependencies of the decay rate and the amplitudes (Figure 13) show that the fast mode decreases its rate considerably and become dominant as temperature approaches the stability limit while the slow mode decreases its rate gradually and disappears. The fast and slow modes surely correspond to the concentration fluctuation and the presence of associates, which are characterized with the intensities $I_{\mathrm{m}}$ and $I_{\mathrm{a}}$ in the static light scattering, respectively. These correspondences between static and dynamic scattering are also seen in the $q$-dependencies of the decay rates and amplitudes. The decay rate of fast mode shows $q^{2}$-dependence while that of slow mode shows a stronger $q$-dependence than $q^{2}$. (Figure 14a) The fraction $I_{\text {slow }} / I_{\text {total }}$ is decreased with increasing $q$. (Figure 14b) (See also Figures 10 and 11 for static light scattering.) These $q$-dependencies indicate that, in this temperature- and $q$-ranges, the corre- 


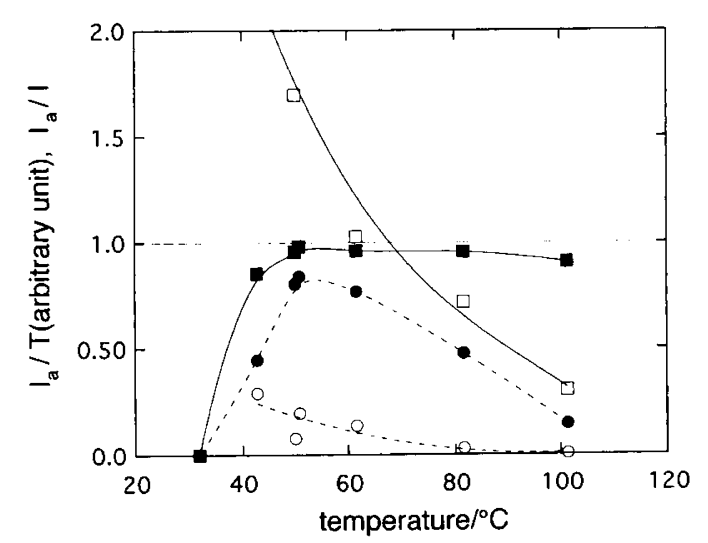

Figure 11. Temperature dependencies of the intensity $I_{\mathrm{a}}$ and its contribution to the total intensity $I$. Curves are guides for the eye. $I_{\mathrm{a}} / T: q^{2}=6 \times 10^{-5} \mathrm{~nm}^{-2}(\square)$ and $4 \times 10^{-4} \mathrm{~nm}^{-2}(\bigcirc) ; I_{\mathrm{a}} / I: q^{2}=6 \times$ $10^{-5} \mathrm{~nm}^{-2}(\square)$ and $4 \times 10^{-4} \mathrm{~nm}^{-2}(\mathbf{O})$

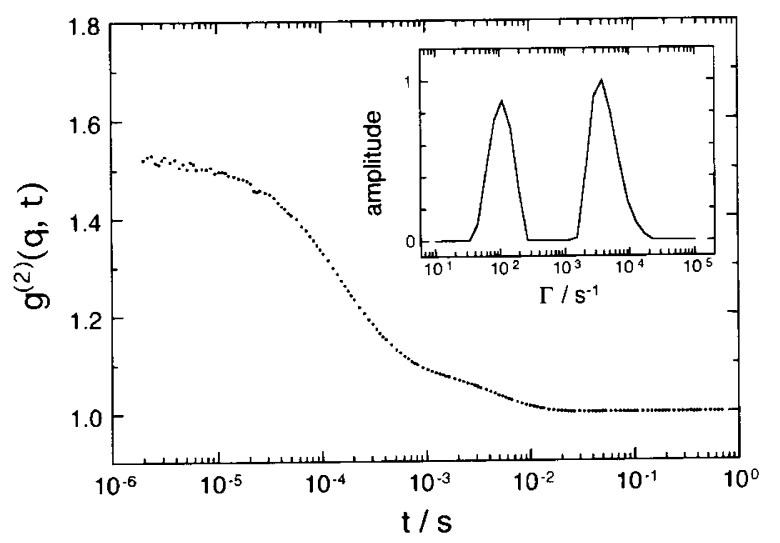

Figure 12. Normalized autocorrelation function $g^{(2)}(q, t)$ of scattered light intensity measured at $\theta=90^{\circ}$ and temperature $=50^{\circ} \mathrm{C}$ for the $15.6 \mathrm{wt} \%$ PS- $b$-PDMS solution in the $40: 60 \mathrm{wt} \% n$-octane+ cyclohexanol mixed solvent. The inset represents the decay rate distribution obtained from the Laplace inversion routine CONTIN.

lation length $\xi$ of concentration fluctuation is not so large yet satisfying $\xi<1 / q$, while the size of associates is rather large, much larger than $1 / q$.

To see effects of the association on dynamics of the critical concentration fluctuation, the diffusion coefficient $D=\Gamma / q^{2}$ for the concentration fluctuation (the fast mode) are plotted against the reduced temperature ( $T$ $T_{\mathrm{s}}$ ) / $T$ in $\log -\log$ scales in Figure 15. The plots show that the apparent critical exponent is around 1.3, which is much larger than that ( $\nu=0.63$ in eq 6 ) for ordinary fluids, while the critical exponents for the static properties have usual values as mentioned before (Figure 8). This must imply that this block copolymer system is somewhat different from ordinary fluids in critical dynamics owing to the presence of associations of diblock copolymers. The exponent of diffusion coefficient different from the usual value seems to indicate coupling of concentration fluctuations and associations, which may be emphasized to be observable in dynamic properties. Details of critical dynamics, e.g., whether the fluctuation and the association are really coupled and how much they are affected each other, will be discussed with experimental data of shear viscosity and tracer-diffusion coefficient of
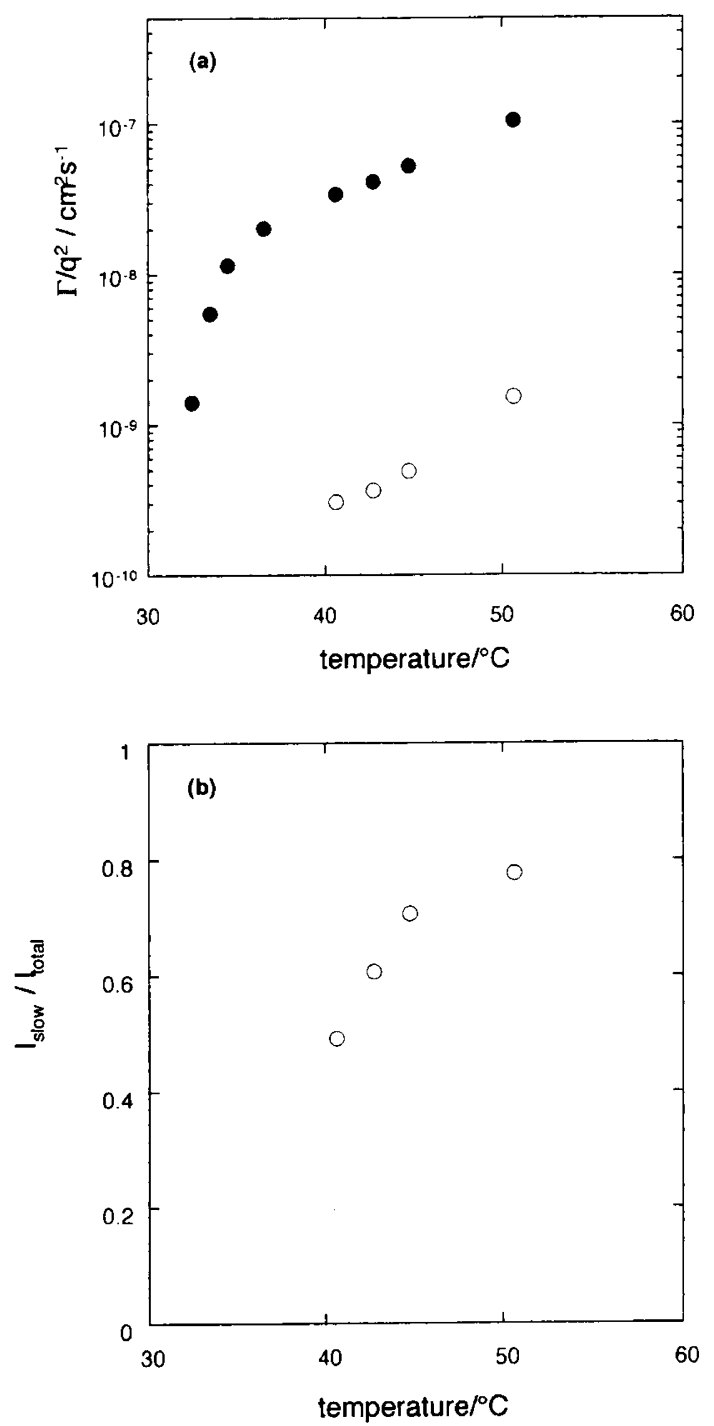

Figure 13. Temperature dependencies of the decay rates and amplitudes for fast and slow modes of the $15.6 \mathrm{wt} \%$ PS- $b$-PDMS solution in the $40: 60 \mathrm{wt} \%$-octane+cyclohexanol mixed solvent at $\theta=30^{\circ} \mathrm{C}$. (a) the reduced decay rates $\Gamma / q^{2}$, and (b) amplitude ratios $I_{\text {slow }} / I_{\text {total }}$.

copolymers in the forthcoming paper.

\section{CONCLUSIONS}

The static and dynamic light scattering study of solutions of block copolymers consisting chemically similar as well as dissimilar blocks in a weakly-selective poor solvent has revealed the following behaviors. The solution of copolymer consisting similar blocks behaves like an ordinary mixture of fluids, show a usual liquid-liquid phase separation, and follow the 3D Ising model near the critical point. On the contrary, in the copolymer solution of dissimilar blocks, two modes are clearly seen in static and dynamic scattering, showing an increasing association as well as concentration fluctuations with approaching the liquid-liquid phase separation. Near the stability limit, the critical concentration fluctuation eventually dominates over the association in light scattering. The critical behavior follows the 3D Ising model as usual fluids in static properties, whereas the critical 

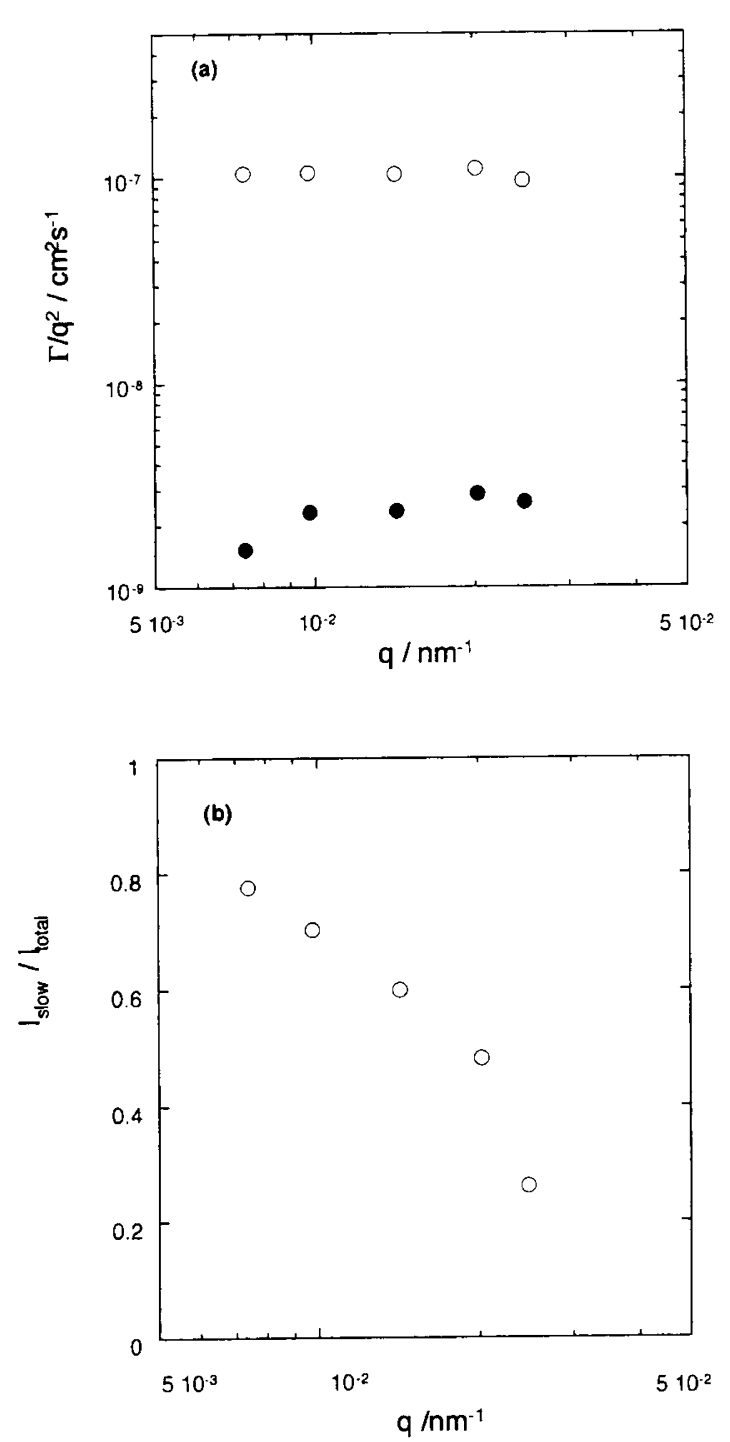

Figure 14. $q$-Dependencies of the reduced decay rates and amplitudes for fast and slow modes of the $15.6 \mathrm{wt} \%$ PS- $b$-PDMS solution in the $40: 60 \mathrm{wt} \% n$-octane+cyclohexanol mixed solvent at $50.63^{\circ} \mathrm{C}:$ (a) the reduced decay rates $\Gamma / q^{2}$ for fast $(\bigcirc)$ and $\operatorname{slow}($ modes, and (b) amplitude ratios $I_{\text {slow }} / I_{\text {total }}$.

dynamics is affected more seriously by the copolymer associations.

Acknowledgments. The authors very much appreci-

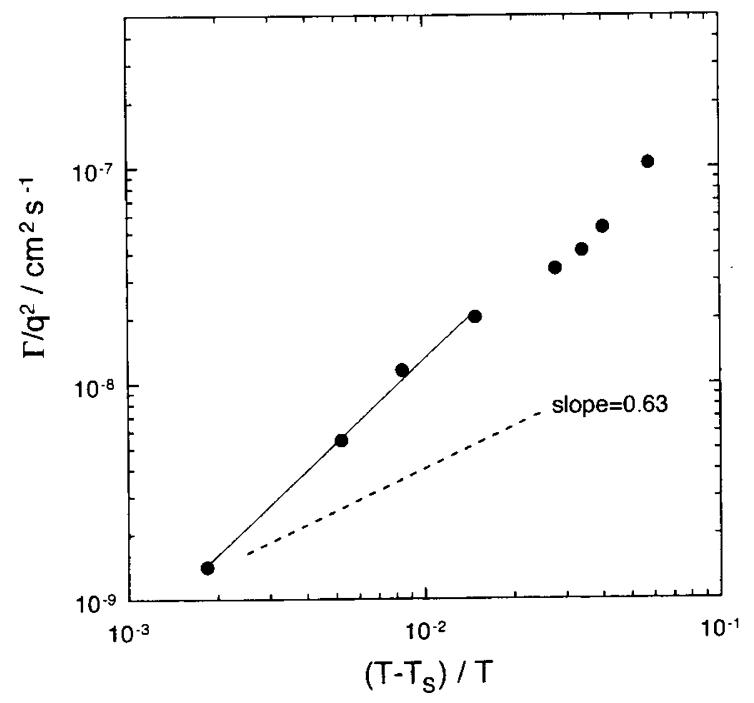

Figure 15. Log-log plots of the diffusion coefficient $D=\Gamma / q^{2}$ against $\left(T-T_{\mathrm{s}}\right) / T$. Solid straight line indicates the least-squares fitting for the 4 data points closest to $T_{\mathrm{s}}$.

ate the preparation of PDMS- $b$-PTMDSE sample by Dr. Atsushi Takano and Prof. Yoshinobu Isono of Nagaoka University of Technology.

\section{REFERENCES}

1. S. Sakurai, T. Hashimoto, and L. Fetters, Macromolecules, 29, 740 (1996)

2. T. P. Lodge, C. Y. Ryu, I. W. Hamley, J. P. A. Fairclough, A. J. Ryan, and J. S. Pedersen, Macromolecules, 29, 5955 (1996).

3 Z. Tuzar and P. Kratochvíl, in "Surface and Colloid Science," E. Matijevi, Ed., Plenum Press, New York, N. Y., 1993, Vol. 15 , pp $1-83$.

4. A. Sikora and F. E. Karasz, Macromolecules, 26, 177 (1993).

5. D. F. Siqueira, S. P. Nunes, and B. A. Wolf, Macromolecules, 27, 234 (1994)

6. J. S. Higgins, S. Blake, P. E. Tomlins, S. B. Ross-Murphy, T. Slaples, J. Penforld, and J. V. Dawkins, Polymer, 29, 1968 (1988).

7. H. Li, G.-E. Yu, C. Price, C. Booth, E. Hecht, and H. Hoffmann, Macromolecules, 30, 1347 (1997).

8. T. Nose, Polymer, 36, 2243 (1995).

9. A. Nagasawa, K. Inomata, and T. Nose, to be published

10. R. Pecora, "Dynamic Light Scattering," Prenum Press, New York and London, 1985.

11. H. E. Stanley, "Introduction to Phase Transition and Critical Phenomena," Clarendon Press, Oxford, 1971, Ch 10, p 170. 